\title{
Analysing the visual dynamics of spatial morphology
}

\author{
Alasdair Turner \\ Bartlett School of Graduate Studies, University College London, 1-19 Torrington Place, \\ London WC1E 6BT, England; e-mail: a.turner@ucl.ac.uk \\ Received 18 July 2002; in revised form 10 November 2002
}

\begin{abstract}
Recently there has been a revival of interest in visibility analysis of architectural configurations. The new analyses rely heavily on computing power and statistical analysis, two factors which, according to the postpositivist school of geography, should immediately cause us to be wary. The danger, they would suggest, is in the application of a reductionist formal mathematical description in order to 'explain' multilayered sociospatial phenomena. The author presents an attempt to rationalise how we can use visibility analysis to explore architecture in this multilayered context by considering the dynamics that lead to the visual experience. In particular, it is recommended that we assess the visual process of inhabitation, rather than assess the visibility in vacuo. In order to investigate the possibilities and limitations of the methodology, an urban environment is analysed by means of an agent-based model of visual actors within the configuration. The results obtained from the model are compared with actual pedestrian movement and other analytic measurements of the area: the agents correlate well both with human movement patterns and with configurational relationship as analysed by space-syntax methods. The application of both methods in combination improves on the correlation with observed movement of either, which in turn implies that an understanding of both the process of inhabitation and the principles of configuration may play a crucial role in determining the social usage of space.
\end{abstract}

\section{Introduction}

Visibility analysis is an intuitively attractive way to investigate the environment as it seemingly gives one the perspective of the (able-sighted) occupant. It allows us to make rigorous mathematical statements about systems, and thus it would appear to allow us to apply mathematical certainty to the experience of urban and building environments. We might use visibility analysis to talk about morphological properties of the built environment, or to talk about how people can move or interact within the visible space, or to discover the significance of objects placed within that space. Visibility analysis has a long history. Thiel (1961) first tried to analyse explicitly the visual properties of spatiotemporal paths through the built environment; Benedikt (1979) looked at isovist measures of visible space throughout configurations and the associated visual fields through space that they produce. Recently there has been a renewed interest in visibility analysis, from examination of the visual properties of routes people actually take (Conroy, 2001), or might take (Lee and Stucky, 1998), to comparisons of visual properties with aggregate behaviour (Desyllas and Duxbury, 2001; Turner and Penn, 1999) and classification of urban types (Batty, 2001). Looking at the historical development of visibility techniques, there is an obvious gap in the application of mathematical analysis techniques as, it would appear, humanist, phenomenological, cultural, and Marxist approaches to geography and similar postmodern approaches to architecture became dominant. Stemming from the work of Harvey (1973), these approaches argue that any spatial analysis of a city (or a building) must relate to its sociological function at some level and, because the sociological function involves many inseparable variables, analysis of pure spatial form is all but meaningless. We cannot access the qualia relating to the personal experience of a space, and thus any attempt at a phenomenological theory of the city or of building is flawed. Even our 
methods of analysis are based on some culturally bound attraction of what we consider important. The combined effect is that we cannot state objectively that one plan or one design is better, sociologically or experientially, than another. We might call this the 'postpositivist' argument.

So why has there been a revival of interest in visibility analysis? Many assume it is a result of the easy availability of powerful desktop computing in the form of geographic information systems (GIS). Postpositivists suggest that this availability can easily lead to an unthinking reliance on the computer, akin to the reductionism of the 1960s (see, for example, Soja, 2001). However, when one surveys the visibility analysis literature it becomes clear that the background of many researchers is very different, and that many are well aware of the postpositivist position. Their solution has been to build their analysis around the psychololgical theories of Gibson (1950; 1979), where the occupant's understanding of the environment is placed outside the model, and instead his or her ecological relationship with the environment is considered through a set of affordances representing these relations; that is, the occupant is engaged in direct perception of the environment. Hence, the relationship is investigated as a humanspatial variable that is differentiable from the social context and individual experience. However, although ecological perception is cited as a source of inspiration by authors, in practice research often tends to be marred either by the view that it might still be possible to provide insights into the phenomenological understanding of architecture, or, by concentrating only on the visible Cartesian space or other formalised extrahuman morphology, a failure to regard properly the ecological relationship of individual and the environment.

The argument advanced in this paper is that, if we are to make a priori predictive theories about the sociological effect of the built environment, we need to comprehend the entire ecological process in action. That is, we need to see perception as an active or dynamic process occuring between the agent and the environment, allied to with the biological notion of autopoiesis (Maturana and Varela, 1980). The importance of regarding the process, rather than formal attributes, of visibility alone cannot be understated. A formal assessment of visibility in vacuo leaves us without a route to gain access to the experiential, societally bound, actions of the individuals within space as the human-spatial variable has been separated. By regarding the process, we still have access to a physical observable variable (the individual, or aggregation of individuals does something in the world); however, it is an observable variable that can be transferred to the level of social interpretation through discussion of the implications of the observed process. In practical terms, this might be: the process leads to a crowd (physical); the crowd is a problem (social). That is, the process can determine the natural outcome of a given configurational organisation. There is evidence from cognitive science that the natural outcome tends to be similar to the actual outcome, regardless of subjective input, that is, that the process is a product of the task to which the organism relates, rather than the evolution or nuture of the organism (Dale and Collett, 2001). In architectural analysis, the possibilities of such an approach are just becoming apparent.

To date, there have been a couple of quantified applications of this process-based theoretical framework to the built environment; Turner and Penn (2002) compare the numbers of people moving through the Tate Britain Gallery with the numbers of agents progressing through an agent-based model of the same environment; Penn and Turner (2002) apply the same agents to a department-store scenario. The agents use natural movement rules through selecting a location at random from the current field of view, and then moving towards it for a number of steps before redefining the destination. Herein, the same agents are applied instead to an urban environment. 
It is found that, unlike the building case, where the entrance is clearly defined, the urban scenario requires careful selection both of 'arrival zones' and of length of time in the system in order to correlate closely with pedestrian movement patterns. However, once these parameters are selected, the technique performs considerably better in predicting human-movement patterns than the use of raw visibility-analysis values based on visibility-graph methods: application of isovist area (after Desyllas and Duxbury, 2001), and isovist (or visual) integration (after Turner et al, 2001). That said, the space-syntax method of axial-line integration (Hillier and Hanson, 1984) correlates as well with human movement as the agent-based approach. Both axialline and agent methods correlate well with each other, but combined together provide a better 'postdiction' of human movement than either alone. It is evident that if axialline integration were working on some visual artifact, then visual integration should also provide a good correlation with human movement-but it does not. Therefore, we are led to the conclusion that axial-line analysis picks out a topological relationship not present either in isovist-based or in agent-based methodologies. As Hillier (1996) puts it, space itself may be the machine, embedding the visual process within the spatial morphology. This has a significance impact on our analytic methods: it suggests that, rather than increasing complexity to an impossible extent to capture all visible facets of the true environment, we may merely need to determine the humanly accessible topology as invoked through the process of inhabitation, thus simplifying the tools an architect or planner requires to assess the social adequacy of a project-at least in an urban context.

This paper comprises five further sections: a detailed review of visibility-analysis techniques, a theoretical basis for the process-based model, experimental results, discussion, and a brief conclusion in which I reflect on how the visual dynamics of spatial morphology might relate to ideas as diverse as surface networks and hermeneutical dialogues.

\section{A background to visibility analysis}

It seems natural to develop tools that can help us understand the experience of the environment, either to help modify or to help improve the experience or the environment itself. Such a motivation led Thiel (1961) to propose a sequence notation of architecture, whereby the details of the experience can be recorded can be recorded for paths as they progress through buildings or an urban environment. The experience is codified by a linear timescale against which symbols denote visible values of the experience drawn from Lynch's (1960) 'imageable' elements (for example, paths, edges, or districts). Thiel evokes Goethe in his description of the enterprise:

"Architecture may well be 'frozen music' like a phonograph record; but man is the pickup whose movement realizes the experience" (1961, page 33).

He views the spatial experience as a biological function, by thinking about the ecological relationship between human and environment inherited from an understanding of Gibson (1950). However, the concept of the meaning of the experience is central to Thiel's recording and, although he fully realises that different cultural backgrounds will play a part in the perception of the experience, he attempts to classify the public conception' of the experience: involving circulation, use, association, expression, and philosophy. Crucial to this public experience is the semantic quality of the environment at a societal level, because, he states, the calibration of the experience would be useless without considering the meaning of signs and symbols within the environment. Thus, society and space are intimately bound to Thiel's methodology at the phenomenological level. 
Thiel provides one thread to the development of visibility-analysis techniques. The other is introduced by Benedikt (1979): he finds that an analysis such as Thiel's lacks a tool that can adequately describe architectural form and space as visually presented. The notation used by Thiel is a set of symbols indicating some categorisation of a continuous experience somewhere between a 'vague' (an environment consisting of a multiplicity of objects) and a 'volume' (an environment defined by surfaces). Benedikt tries to find a more precise way to think of a space by introducing isovists to architectural analysis. An isovist is "the set of all points visible from a given vantage point in space and with respect to an environment" (1979, page 47). Benedikt constructs an isovist by taking a two-dimensional slice through a polyhedron bounding the volume visible from a particular point in space [see figure 1(a)]. He demonstrates various measurements that may be taken of these isovist polygons, such as the area, perimeter, circularity, and so on. The values of these may be plotted as contour lines on a building or urban plan to show how the value varies across the space [figure 1(b)], creating an 'isovist field'. The importance of these contours, Benedikt suggests, is twofold: first, they may be of use in the study of behaviour and perception; and second, they may be of use to identify architectural archetypes. The inspiration behind the perceptual quality of isovist fields is Gibson. Gibson (1979) introduces the concept of optic flow, which may guide an individual through a landscape; in fact, he sees movement as essential to perception of the environment, in that there is no ecological phenomenon without the interaction of the occupant with the environment. Thus Benedikt suggests that it is the rate of change of the isovist field which may be of use in investigating perception and behaviour. When contour lines are closely packed they suggest a rapidly changing view, and the behaviour of the occupant might be either to take a shallow path around the rapid change, or to slow on approaching it in order to take in the new vista. With regard to the identification of archetypes, Benedikt suggests

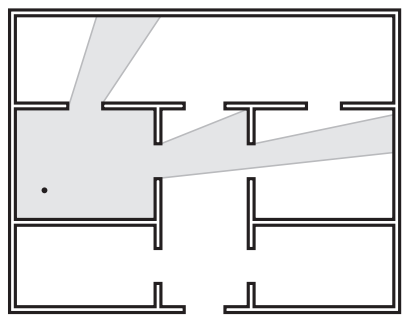

(a)

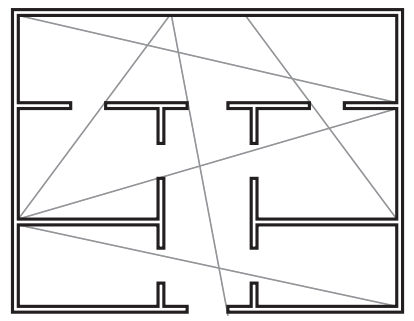

(d)

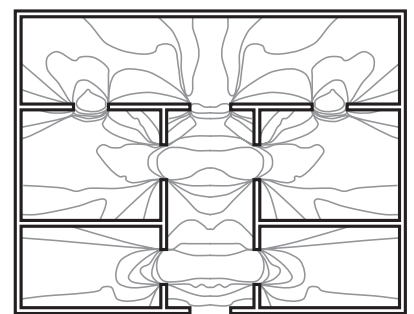

(b)

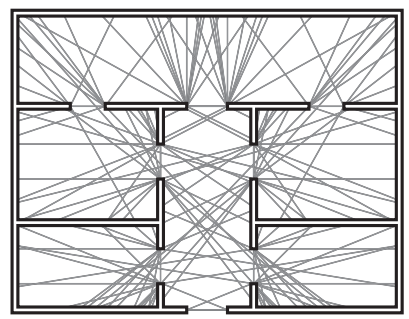

(e)

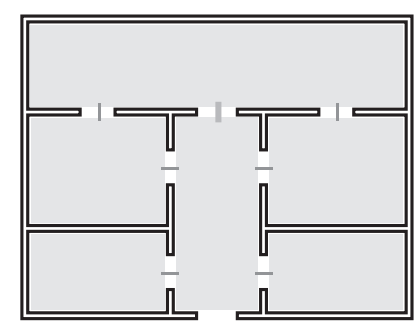

(c)

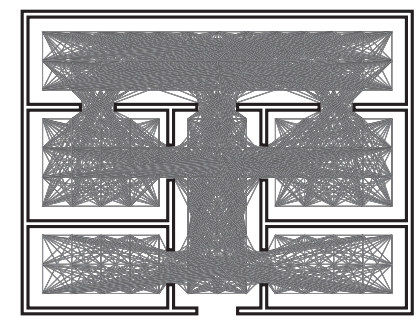

(f)

Figure 1. (a) An isovist polygon is a two-dimensional slice through the volume visible from a location. (b) An isovist field of isovist area provides contour lines of equal visible area. (c) The set of convex spaces for a configuration. (d) A set of axial lines (the central line connects the system to the external environment). (e) The e-partitions produce areas where the number of visible surfaces does not change. (f) A dense grid visibility graph records the connections between mutually visible locations, which may then be used for graph analysis. 
that there may be common features of isovist fields that can be used to define ('in good part') kinds of space, such as 'hall', 'corridor', 'colonnade', and so on, by looking at typical values the analysis provides across the space. Such classification are still of interest to researchers today. Batty (2001) provides a comprehensive set of isovist analyses at the building, urban, and town scales, looking at how the isovist properties vary within them. He also revisits the idea that one might be able to reduce isovist descriptions of large regions significantly by finding a suitable covering set of isovists. Davis and Benedikt (1979) prove that it is possible to obtain a small sufficient set of isovists that cover an open space fully; Batty demonstrates an algorithm which constructs a sufficient set of isovists by selecting the largest isovist first followed by successively smaller ones to cover a building environment.

The tools of visibility analysis are almost all derived from Thiel and Benedikt and, although there have been applications of isovists and sequence notations, until recently there have been few advances in the tools used for visibility analysis itself. For example, isovists have been used to investigate aspects of architecture. Hanson (1994) applies isovists as well as space-syntax techniques to 'deconstruct' several well-known architects' houses. She examines the way architects have composed space, much as Benedikt looks at representative values of isovist fields for different styles of architecture. Similarly, Benedikt and Burnham (1985) went on to consider further the effect of the properties of isovists on perception, finding that perceived values of 'spaciousness' related to the complexity of the isovist rather than to the area of the isovist. However, although there has been little advancement in visibility-analysis tools, there has been continuous development in the intimately related area of morphological analysis. For example, the morphological components used by March and Steadman (1971) to generate graphs of building elements are 'rooms' or 'corridors', which, although distinguishable spatially, also fit into the notional units of visual space as introduced by Thiel, who talks in terms of 'districts' and 'paths'. These visual units overlap the morphological ones more clearly when graphs of urban elements are considered, as, for example by Krüger (1979). Space syntax (Hillier and Hanson, 1984) formalises these elements into convex spaces [the near-minimal set of 'shortest and fattest' convex polygons covering the space-see figure 1(c)] and axial lines [the set of fewest longest lines passing through the convex spaces that retain the topology of the systemfigure 1(d)]. Once again, the lines may be considered as paths and the convex spaces as districts, and indeed axial lines are often referred to as 'the longest lines of sight' through a configuration. Furthermore, space syntax focuses on the relational aspects of elements, and the relationships have parallels with Thiel's spatiotemporal paths through the environment. The depth ${ }^{(1)}$ from one element to another, in terms of number of convex spaces passed through or axial lines turned through, represents a sequence of transition through the environment, relating to one possible experience of the environment. The mean depth is the average depth from one element to all other elements in the system, and essentially reflects an enumeration of all possible sequences through the building or urban environment (in terms of spaces and lines), and thus

(1) In terms of graph theory, we can consider a graph $G(V, E)$ as the combination of a set of vertices $V$ (in this case, the convex spaces or axial lines) and a set of edges $E$ consisting of edge pairs $\left\{v_{1}, v_{2}\right\}=e_{12}$ joining the vertices (in this case, convex spaces adjacent to one another, or axial lines crossing each other). A path $P$ between two vertices $v_{1}$ and $v_{2}$ can be defined as a sequence of edges $e_{i j}, e_{j k}, \ldots$ from $E$ that is either simply $e_{12}$, or starts with $e_{1 n}$ and ends with $e_{m 2}$, containing further edges from $E$ subject to the condition that $e_{i j} \in P \Leftrightarrow e_{j k} \in P$ and $e_{i j}$ is the immediate predecessor of $e_{j k}$ in $P$. The 'depth', or shortest path length $L$ between the two vertices, is the number of edges in the shortest constructable path between $v_{1}$ and $v_{2}$. 
might be considered to give an idea of what the general experience of a space might be from this location.

Other measures of morphology are more explicitly related to visibility. Peponis et al (1997) introduce the notion of e-partitions and s-partitions. An e-partition is an imaginary line such that if (and only if) the occupant crosses it a vertical planar surface either comes into view or is lost from view. Algorithmically, the set of e-partitions can be constructed from a plan by drawing the extensions of the surfaces that end in reflex corners (internally convex corners), and the extensions of the imaginary lines that join corners to reflex corners [figure 1(e)]. S-partitions are the subset of e-partitions that extend surfaces at reflex corners, for examples, as one passes the end of a building. E-spaces are defined as the regions between these partitions. Provided there are no curves to consider, this leads to a unique partitioning of the space into informationally stable' units, that is, spatial units where the number of visible surface does not change. E-spaces have further useful visual properties, such as the fact that a sufficient set of isovists may be constructed simply by locating one isovist for every e-space. Peponis et al suggest using them for the determination of experience of the space, and for further relational analysis of the way the e-spaces are connected together.

Recently, there has been a resurgence of interest in visibility for use in architectural analysis. In part, we can observe the influence of geoinformation science (GISci), which has concerned itself with viewsheds. Viewshed analysis tends to focus directly on the relationship of the occupant to the environment by including a viewer height, and originates from landscape studies by Amidon and Elsner (1968) and Lynch (1976). The viewshed is the set of all visible ground points from the viewer's location. The field of GISci has refined techniques for viewshed determination over the years, investigating the effect of the accuracy of the underlying data (Fisher, 1991) and the generation of 'fuzzy' viewsheds (Fisher, 1995). Viewshed analysis has also spawned new techniques for investigating the experience of the landscape environment. Llobera (1996) uses differential properties of viewsheds to determine a measure of the enclosure of the environment. Lee and Stucky (1998) discuss the visual characteristics of paths through the environment, combining the idea of isovist measures explicitly with a sequence notation, in order to produce paths with, for example, maximal visibility of the surroundings which might provide walkers with pleasant views. In architectural work there has been a resurgence of visibility techniques with advances in agent-based simulation and virtual reality (VR) technology; Penn et al (1997) have developed agents that roam VR environments to retrieve isovists, and Batty and Jiang (1999) have developed a parallel agent system to construct isovists. Extending work on isovists, Conroy (2001) has gone on to investigate the formal visual properties of paths that people take within controlled experiments in VR environments. She finds that people pause at 'junctions' (among other correlates such as high isovist area and long lines of sight), and therefore attempts to classify 'junctionness' through measures of the isovist. Conroy or Lee and Stucky's notion that isovists might be chained together in a path leads to the realisation that isovists may be connected into a graph across a surface, and indeed Conroy defines 'syntactic' isovist arrays. Turner and Penn (1999) have arranged such arrays of isovists on Euclidean grids, and construct connections between them if their centres are intervisible or if the isovists overlap. They use the resulting graphs to calculate the visual depth from one location to all other locations within the system (calculated as per the space syntax graph measure of depth). The visual depth defines a condensed Thiel sequence notation: a measure of the outgoing visual area on any path from a particular location. Turner et al (2001) show that an isovist graph is more correctly a form of visibility graph. That is, it is simply the connection of all mutually visible points across a dense grid [see figure 1(f)]. 
They investigated various graph measures, including mean shortest path length (or mean depth), which corresponds to the average number of turns from one location to any other within the system, as well as other measures from graph theory. Thus, visibility analysis is linked into a corpus of literature on visibility graphs - from their practical application in the location of services [for example, Braaksma and Cook (1980) looked at the placement of airport facilities, and De Floriani et al (1994) examined optimisation of the placement of radio transmitters] to their theoretic application to mathematical problems such as the museum-guard problem (see O'Rourke, 1987). Turner et al suggest that the dense grid graph properties may be "closely related to manifestations of spatial perception, such as way-finding, movement, and space use" (2001, page 103), and this is backed up by further research by Desyllas and Duxbury (2001), who find a correlation between movement along pavements and measures of the visibility graph.

From this discussion, it is noticeable that visibility analysis involves some form of one-dimensional sequence notation or two-dimensional isovist measure. FisherGewirtzman and Wagner (2002) point out that to be complete, visibility analysis really should cover and quantify the third dimension as Benedikt managed to do for two dimensions. They suggest a three-dimensional analysis of 'spatial openness' (or isovist volume) of views from flats, although because of computing processing power, they too restrict themselves to two-dimensional isovist area. In order to resolve the difficulty of computational complexity Teller (2003) takes a novel solution to three-dimensional analysis. He investigates the angular extent of the sky visible throughout the urban environment, to calculate the 'sky opening' from locations. This provides a scaleinvariant metric of the environment, which Teller offers as a useful way to avoid the scale dependency of many measures of isovists - such as area or visual integration. $\mathrm{He}$ proposes that this will be useful for classifying town squares and also for examining the effect of new buildings on the perception of the environment, and provides examples of typical values. A similar approach is demonstrated by Ratti (2002), who plots distributions of visible sky area from different locations in order to give a weighted directional measure of visual openness. Although these innovative techniques add a three-dimensional quality, they do not break down into new uses of visibility analysis, which can be split essentially into two categories: either classification or assessment of the perceptual qualities of the environment. That is, every investigation is either the quantification of the experiences offered (by the architect) or received (by the occupant).

\section{Modelling the visual process of inhabitation}

Visibility-analysis tools are produced, of course, in the hope that they might be useful. Useful, for example, to determine what the perceptual qualities of a building might be, or to categorise different urban types. It is important to realise that both these forms of usefulness are only meaningful in relation to a social variable. In the case of categorisation, what we designate a 'space' itself is loaded with our cultural interpretation of space, so that even labelling a 'space' as a 'room' or a 'street' depends on what our understanding of a room or a street is. A 'room' or a 'street' are both defined via comparison to the researcher's social framework, not to her or his spatial framework, and thus she or he encounters what Harvey (1973) refers to as 'individuation' in the different languages of spatial and social variables. When the researcher goes further to assess the 'experience' of a space, the problem is compounded by the requirement to project our understanding of the experience onto the occupant of the space, who in turn has his or her own subjective understanding of the space. These objections may only seem to be relevant to earlier analysis, for example, when Thiel (1961) suggests that it is possible to derive a 'public conception' of experience, or when Benedikt (1979) 
suggests that it might be possible to extract the features of a 'colonnade'. Today, it would seem, we have overcome the errors of our ways. However, it is easy to fall into positivist thinking. If we look at a feature of visibility analysis, we may want to say something such as this location is more 'strategic' or that location is more 'accessible'. The words 'strategic' and 'accessible' seem at first objective, but if we think for a moment we realise that they are linked to physical and social preconceptions of what 'strategic' and 'accessible' are. Accessible to a pedestrian may not be accessible to a wheelchair user, or to someone with crutches. Our perception of 'accessible' also depends on the social or political context, gender, ethnicity, and any number of postmodern or poststructural concerns about the background of the occupant involved. These arguments have a direct bearing on Llobera's (1996) use of the word 'enclosure', a measure of the differential properties of viewsheds, or the space syntax use of 'control' or 'integration', or when Batty (2001) tries to find 'signatures' of urban types, or Thiel (1998) applies visibility analysis to access a phenomenological variable.

Hence Benedikt (1997) and Soja (2001) find it necessary to reiterate the postpositivist position. Soja writes:

"Problems arise when accurate descriptions are projected too far as explanations of social behaviour, or as primary factor shaping the phenomenology of spatial experience of everyday life, or as foundations for a general theory of the city" (2001, page s1.3).

So what is to project an accurate description too far as an explanation of social behaviour? Conroy (2001) observes people moving through the environment and realises that they slow down at what, to her, seem like junctions (as well as noting other correlates). Therefore, she produces a visibility-based variable of junctionness, and determines whether or not it correlates with how the people move. We should emphasise that this as, it stands is good scientific procedure. To project too far is to go further than Conroy and to ask the question: what does this mean for how people move through spaces? We cannot suggest people slow because there is a junction (or a degree of (junctionness'), as there might be an underlying variable we have not uncovered. This is the general problem of underdetermination, or ecological fallacy. If we are to make a predictive model, however, then we will need to make assumptions about the way people are acting in the world. ${ }^{(2)}$ It would appear that we require a causal chain which cannot evade the inclusion of a social variable. For example, a model which uses 'junctionness' as a causal variable will be rendered useless if the response to 'junction' is simply a cultural artifact. Thus, in order to avoid projecting an accurate description too far as an explanation of social behaviour, we need to answer the question: how do we make a model whose applicability is unaffected by the presence of an unknown social variable?

It seems to me that we should consider how society enters our models in the first place. For, if we try to write down the physical process of inhabitation, the thing we call 'society' is absent. There is a set of individuals, each with their own belief system, which interact physically with the environment and with each other. The belief system is modified only by these interactions. Figure 2(a), which borrows heavily from Maturana and Varela (1987), shows such a system. The circles represent the fact that the individual's thought processes are a closed loop, which is modified only through mediated interaction with external influences. The influences themselves are physical interactions, and thus are observable entities limited by the physicality of the agents (or individuals) involved (for example, the ability to produce coordinated sets of noises, or speech). 'Society' in figure 2(a) is something that happens somewhere in the individuals and that

(2) Oreskes et al (1994) offer a concise account of problems that affect models is general. 


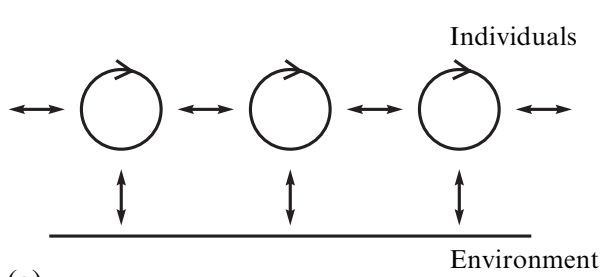

(a)

Environment

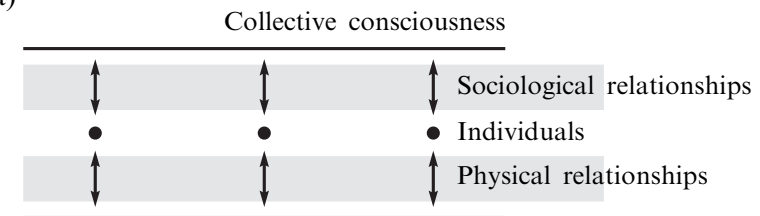

(b)

Environment

Society

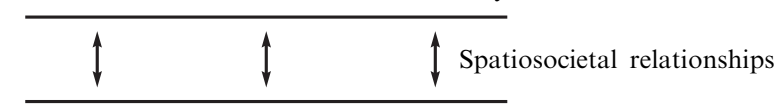

(c)

Environment

Figure 2. Abstractions from individual to society lead to the positivist crisis. (a) The physical system: individuals are 'closed' systems that interact with the environment through physical processes. (b) Collective consciousness is abstracted from the individuals. (c) Removing the layer of the individual results in the loss of independence of social and spatial variables.

leads to coordinated actions, but we cannot grasp it as such. To include the notion of society, we need to abstract out from the physical situation. Figure 2(b) shows such an abstraction, where the common knowledge shared by the individuals has been squeezed out into a collective consciousness. Notice that this abstraction is upwards from the individual. An ardent poststructuralist might claim that this is incorrect, that society pervades the whole of the system: surely it is written into the sands. However, this is to deny that society is a living entity. The actual physical results of society are relics of (possibly instantaneously) previous societies, and the relics result from physical actions of the individuals which comprise the society. We can argue about the nature of the upwards abstraction - for example, is it closely or weakly bound, a process of dialectics or of structuration-but these questions can be clearly delimited for sociologists to discuss. What is important is that it does not actually impinge downwards into the physicality of interaction with space. We can thus separate the diagram into two distinct phases: one of sociological relationships and one of physical relationships. Although the relationship of the individuals to 'society' may be less well defined, the relationship of the individual to space remains well defined observable processes. The crisis of positivism strikes not at this level, but when we come to abstract further to exclude the individual. Figure 2(c) shows how spatial and social variables may easily become mixed when the individual layer is removed, inviting postpositivist criticism.

If we remain at the level of abstraction shown in figure 2(b), we can examine the natural behaviour of that functioning body in relation to its environment in order to understand the natural behaviour of an aggregation of agents, without effect from the social layer of collective consciousness. Soja (2001, page s1.4) claims that it can be "highly misleading to think of a physical influence as an independent factor or relation in attempting to understand the complex interplay between spatial and social phenomena", whereas to treat physical influence as an independent factor is precisely what this method is intended to do. We have to realise that the social layer has not been removed, and that it will influence behaviour; however, on this fundamental microscopic level, it operates in an 
entirely different manner to the physical layer on how the being operates. That is, although the walking occupant is deterred by a no entry sign, the physical manner of her or his walking itself is unaffected. ${ }^{(3)}$ The physical relationships in a system are bounded by the physical constraints of the organism concerned. If we construct a physical model of the organism, and a physical model of the environment, we should be able to obtain the range of possible interactions. This reads essentially as a statement of autopoiesis, which is the process which maintains the individual or agent within the environment (Maturana and Varela, 1980), albeit at the level of cell biology. Autopoietic theory provides the idea of 'structural coupling', where the agent affects and is affected by the environment, such that they are conjoined in an ecological phenomenon. The combined effect of the structure of the environment and the structure of the agent determine what the agent does. Although this at first seems difficult to reconcile with the idea of a human agent, which has intelligence, thought, and motivation, on certain levels it is trivially true: a $2 \mathrm{~m}$-high wall opposes all but the most determined movement, a chair gives the possibility of sitting on it. That is, provided we leave the intelligence at the level of the collective consciousness, we can look solely at the ecological process of behaviour. The possibilities presented by the environment are affordances (Gibson, 1979). We can implement a simple agent model based on the idea of affordances, by giving the agents and environment a set of affordances to join them. Evidently, if the process under observation is the natural process of movement, perception only exists if there is movement to enable it, that is, an optic flow of information in existence.

In order to establish a process of 'exploratory' movement, in Turner and Penn (2002) we implement agents which have a visual field. Natural exploration is enabled by allowing the agents to pick a location at random from the visual field (that is, all locations have an equal affordance for further exploration). In that paper we suggested that this is the "natural mode to move around an art gallery"; stated explicitly, our particular application was to an 'exploration task'. That we should properly call it a 'task' derives from Wheeler's (1996) argument that what we are in fact doing by setting agents into active or dynamic perception is establishing a hermeneutic dialogue between the agents and the environment. The dialogue itself cannot be accessed directly, but its rules can be allowed to evolve. Essentially, our implementation in Turner and Penn (2002) was at the level of Brooks's (1991) subsumption architecture. In the subsumption architecture, the agent, or 'animat' (in this case a robot) is divided into layers which perform particular functional roles, such as visual input, or motor output. However, the robot is given no knowledge representation; the architecture simply allows a researchers to assess the robot's natural ability to fulfil a task, such as locating itself in the middle of the room. However, Wheeler argues that in order to establish a dialogue properly we have to leave the animat to evolve into its natural, structurally coupled, relationship to the environment, as proposed by Harvey et al (1997). The approach has been implemented in a number of scenarios: most relevant is Dale and Collett's (2001) perceptual evolution of animats which approach a post in an insect-like manner. By varying the physical attributes the animats either approximate observed 'flying' (similar to bees) or 'walking' (similar to ants). Thus, Dale and Collett conclude, it is the physical attributes of the animal that determine how it interacts with the environment, rather than the perceptual evolutionary history, as it is the task boundaries combined with the physical properties of the agent and the environment that set up what the agent or animat will do. When we applied the

(3) A quibble to this formulation might be that 'quaking in one's boots', a sociological phenomenon, does affect the manner of one's walking. The point here is, it does not affect the motion of physics of the legs as levers attached to the human body. The only things that do this are the scars of societal interaction, which at its root is once more physical, such as loss of limbs in a war. 
methodology to architecture, we chose an 'exploration task'. In retrospect, it is not clear that this is necessarily the most sensible task to apply. In an urban environment, as demonstrated here, the tasks will include 'going to work' and 'having lunch'. That is, the social functions around which our model is developed. Note, however, that by applying a dynamic or active perception, we have retained a separation of spatial and social variables. What the agents $d o$ is determined by the physical limitations surrounding going to work or having lunch, not the social practices involved in them.

\section{An application to the City of London}

In this section agents are applied to the task of inhabitation of the City of London. Because the definition of 'going to work' implies the existence of workplaces, and 'having lunch' the existence of places to eat, for the time being the City is treated in the same way as the art gallery: a place to explore. Agents are released from various locations and left to explore for various lengths of time in order to try to reproduce the movement patterns of actual pedestrians in the area. This maintains a simple model, yet is adequate to generate patterns of movement. When compared with data from the Space Syntax Laboratory (1996), it is found that specific release patterns and exploration times are required in order to correlate well with pedestrian behaviour (up to $R^{2}=0.67$ ). The results are further compared with other, static, visibility analyses as well as space-syntax methods. It is found that whereas other visibility analyses are less associated with movement $\left(R^{2} \leqslant 0.48\right)$, axial-line analysis does correlate well $\left(R^{2}=0.66\right) .{ }^{(4)}$ This leads to a discussion of the relative importance of process and configuration in the natural inhabitation of the City, and a multivariate 'postdiction' that gives a correlation coefficient of $R^{2}=0.73$.

\subsection{Agent-based experiments}

In order to construct agents suited to a natural exploration task, an exosomatic visual architecture (University College London, 2000) was used. In this software architecture, an underlying dense grid visibility graph is used to store all visible locations from any particular location. The visible locations are split into thirty-two angular bins, which then can be used to construct approximate fields of view from any location (see figure 3). This allows rapid retrieval of visible locations from the visibility-graph database for many concurrent agents. To define movement from this scheme, an agent is allowed to roam freely - every so often picking a destination as a location randomly chosen from its field of view. To obtain the field of view, the agent's heading is rounded to the nearest bin and its position is rounded to the nearest visibility-graph location. From this information the set of locations comprising the field of view $F$ is drawn from a subset of bins either side of the current bin. To ensure onwards exploration, the agent takes a number of steps towards the destination before reselecting a destination in the same way. In Turner and Penn (2002) we found the best combination of parameters
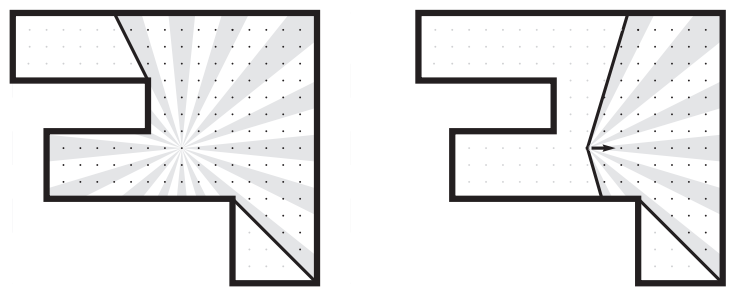

Figure 3. In order to construct a lookup table for approximate fields of view in the agent implementation, locations in a visibility graph are split into thirty-two angular bins.

(4) The axial-line result is reproduced from Space Syntax Laboratory, 1996. 
for the reproduction of human-like movement patterns in a building environment was fifteen out of thirty-two bins $\left(\sim 170^{\circ}\right)$ field of view, with reselection of destination every three steps. For the current study, these best-performing agents were applied to a section of the City of London within a $1.5 \mathrm{~km} \times 1.5 \mathrm{~km}$ area comprising nine OS Landline data grid tiles (figure 4). A visibility graph was produced at $3 \mathrm{~m}$ resolution in order to construct the exosomatic visual architecture for the agents covering the area between building footprints. The arbitrary choice of $3 \mathrm{~m}$ was used in order to keep the visibility graph to a manageable size: approximately 90000 grid locations in open space. For our original studies (applying the agents to an art gallery and a department store), our grid spacing was $0.75 \mathrm{~m}$, determined through human step size. Although it might seem misguided to apply a larger grid spacing than this at present, it appears in the light of the discussion below that the exact spacing does not matter to the theoretical model.

To compare agents with human movement, data from a previous study were used. An area around the Baltic House Exchange site (now the location of the Swiss Re building, designed by Foster Associates) in the City of London was analysed a number of years ago by researchers at University College London (Space Syntax Laboratory, 1996). They conducted a movement study for seventy-eight gates for an approximately $1 / 4 \mathrm{~km}^{2}$ area in the centre of our chosen study area (figure 4), and compared the levels of movement with axial-line integration (after Hillier et al, 1993). For comparison with the agents herein, the observed gate counts were averaged for the day, although the report gives figures for more detailed analyses. Agents were released into the system from random locations within a set of specified regions

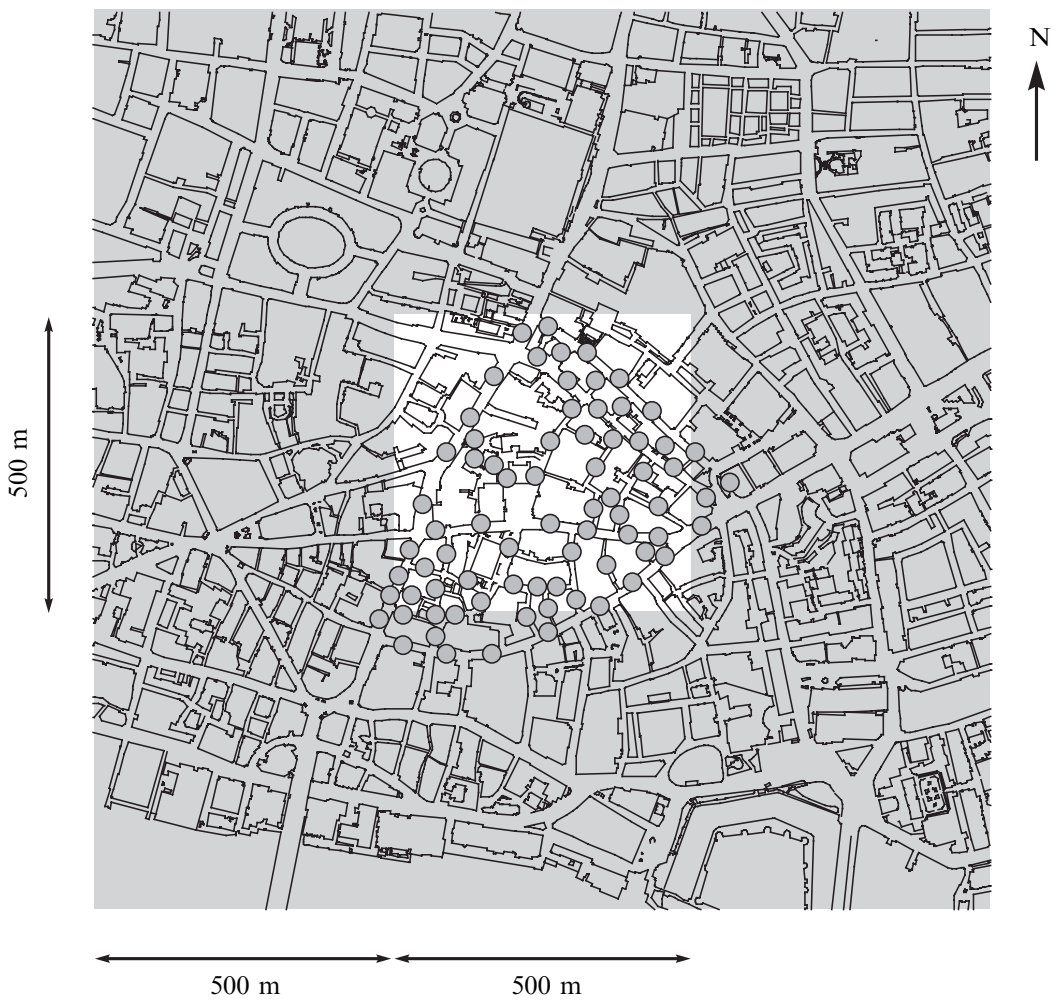

Figure 4. The $1.5 \mathrm{~km} \times 1.5 \mathrm{~km}$ urban area used for the analysis techniques applied here. Gates used for people-movement counts are marked, as well as the approximate $500 \mathrm{~m}$ buffer zone around the central OS map tile. OS Map data (C) Crown Copyright, used with kind permission. 
$R=\left\{r_{1}, r_{2}, \ldots\right\}$, at a constant rate of one agent every fifth timestep. Each agent was allowed to explore for a number of steps $n$ (taking one $3 \mathrm{~m}$ step every timestep) before being removed from the system. Hence the parameter space for the experiments is defined by $R$ and $n$. In order to provide a simple release zone set, a $1.5 \mathrm{~km} \times 1.5 \mathrm{~km}$ release-zone mask was passed across the analysis area from west to east (left to right), and separately from south to north (bottom to top). The number of steps $n$ was varied by a set from 100 (that is, simulating a $300 \mathrm{~m}$ journey) to 500 (that is, a $1.5 \mathrm{~km}$ journey). Gate counts were made in a way exactly analgous to human gate counts, by incrementing the count when an agent passes through a gate zone. When compared with pedestrian gate counts, the agent data required log transformation to give a normal distribution of data points; there is an exponential relationship between the agents and the numbers of people observed. It was found that moving the release mask from south to north made little difference to the correlation with human gate counts, which remained between around $0.45<R^{2}<0.50$ with $n=250$. However, the west to east release made a large difference, with correlation peaking at $R^{2}=0.67$ with $n=250$. Figure 5(a) shows how the correlation varied as the mask was moved. In the case of number of steps, there is a more predictable peak around $n=250$, or $750 \mathrm{~m}$, as shown in figure 5(b).

The fact that the steps correlation peaks at $750 \mathrm{~m}$ seems quite natural given the dense urban fabric of the City - with many public transportation nodes dotted around the area it is hard to walk further than $750 \mathrm{~m}$ without encountering one for onward movement. The transportation nodes may well also be the cause of the other dominant factor: the west to east release-zone bias. When entering or leaving the City, it appears many people use the Underground and mainline stations to the western side of the region around the Baltic House Exchange site. Confirmation of this appearance
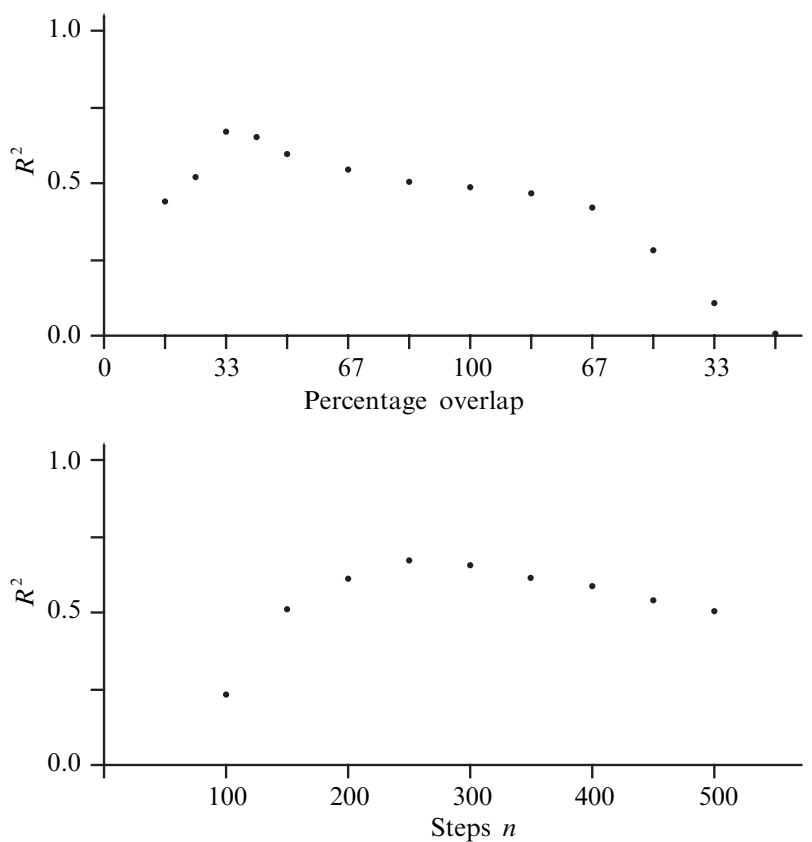

Figure 5. The correlation of agent gate counts with pedestrian gate counts as a release mask is passed across the analysis area from west to east. The $x$-axis scale shows the percentage overlap of the mask with the analysis area. (b) The correlation as the number of steps $n$ agents take within the system before being removed is varied between 100 and 500 . 
is subject to current ongoing research. For now, note that the parameters have a significant effect on the outcome, to which I will return in the discussion below.

\subsection{VGA and axial-line experiments}

Two further sets of analysis were conducted for the urban area to give a comparison between agent method and VGA (visibility graph analysis) graph measures, which it has been suggested may be of use for predicting movement rates (Desyllas and Duxbury, 2001; Turner and Penn, 1999). First, only the pavement areas were included for the analysis, following Desyllas and Duxbury, who suggest this is a more accurate (and more natural) method for the determination of people movement. They correlate VGA neighbourhood size $k$ (which approximates visible accessible pavement area) with pedestrian movement for an area in the City of Westminster, London. In order to repeat the experiment for the current analysis area, a visibility graph of pavement locations (where building footprints were considered to block visibility) was made at $1 \mathrm{~m} \times 1 \mathrm{~m}$ resolution. This yielded about 180000 grid points for analysis. ${ }^{(5)}$ Neighbourhood size $k$ values (approximating isovist area) and mean shortest path length $\bar{L}$ values [or visual integration, as per Turner and Penn (1999)] within this graph were averaged over approximately $25 \mathrm{~m}^{2}$ zones around the gate locations (figure 4) to provide a value to correlate against human movement gate counts. Second, the values were also prepared with the aid of the original full roads $3 \mathrm{~m}$ visibility graph employed for the agent experiment. As with the agent experiments, a better normal distribution of data points was obtained if the results were plotted on logarithmic scales. The data were compared with the observed movement counts, with results shown in table 1 . It is noticeable that the pavements-only analysis is worse than the roads analysis. This might be expected as the area under observation has many different scales employed, from covered pedestrianised courtyards to wide, open, streets. It might also be expected if we again think about the process involved: where a pedestrian chooses a road to move through, it may well be the wider rather than the narrower, despite the pavement width on both roads being equivalent. Another problem may simply be that the data used here are averaged for both sides of the street; Desyllas and Duxbury (2001) note considerable differences of usage between different sides of the street, and had the data been separable, the result may have favoured this technique better.

Table 1. Correlation coefficient for observed people movement gate counts against predicted outcomes.

\begin{tabular}{lllll}
\hline Visibility-analysis model & \multicolumn{4}{l}{ Correlation with observed movement } \\
\cline { 2 - 4 } & $R^{2}$ & $F$-ratio & $t$-ratio & $p$-value \\
\hline Isovist area (pavements) & 0.45 & & \\
Isovist area (roads) & 0.48 & & & \\
Visual integration (pavements) & 0.29 & & & \\
Visual integration (roads) & 0.31 & & & \\
Agent-based model agents & 0.67 & $154(1,76)$ & 12.4 & $<0.001$ \\
Axial-line integration & 0.66 & $148(1,76)$ & 12.2 & $<0.001$ \\
Multivariate agents/axial & 0.73 & $102(2,75)$ & $4.46 / 4.16$ & $<0.001$ \\
\hline
\end{tabular}

(5) This compares with the order of 10000 grid points we were able to analyse in 1999 . The problem of generating visiblity graphs is $\mathrm{O}(N)$ for urban systems as $k$ is small compared with the size of the graph. The $\bar{L}$ measurement used later is $\mathrm{O}\left(N^{2}\right)$ (breadth first search from one point to all other points, for all points). However, the computation time can be significantly reduced as $k$ and $\bar{L}$ only have to be calculated for points within gates. 
Having compared pedestrian-movement counts with the visible area from locations within the gate areas, the data were next correlated against visual integration. This gave a surprisingly poor result, both for pavement-only and for full-road analysis, especially given the previous Turner and Penn (1999) study, where we reported $R^{2}=0.58$ correlation with people moving in a department store. We should note that this earlier published result is for a building rather than an urban scenario and, even within building scenarios, subsequent studies have been concentrated on comparison with occupation rather than movement levels. The result here is poor, and even more obviously poor when compared with the correlation with pedestrian movement for axial-line integration $\left(R^{2}=0.66\right.$, table 1$\left.),{ }^{6}\right)$ which we would initially assume to be achieving results in a similar manner, through calculating the number of turns to another line in the system rather than the number of changes of direction to any other location in the system. It clearly is not.

\section{Discussion}

Batty (2000) argues that there must be a dynamic element to an analysis in order to model what is an underlying dynamic process - in this case, people moving about a city. Initially, this would appear to borne out by the results shown. In particular, that a model without a causal justification, the visual integration, is not associated with pedestrian movement, whereas the agent-process model is. However, if we think of the agents as visual samplers of the environment, there is a marked similarity between the analytic end product of both techniques. In visual integration, values are assigned to a location based on its visual accessibility. In the case of the agents, they approximate a Markov chain operating through locations on the visibility graph. The transition probability from one vertex to another is based on the visual extent in the direction travelled. It would appear, then, that the agents sample visual accessibility as they move through the graph, and more highly accessible locations will be favoured by more agents. However, this explanation ignores the process involved. If the agents were allowed to move freely in any direction then they might be considered such samplers, but in fact, as the transition probability is directional, once they have started to move in a certain direction, they will continue to move in that direction. Certainly, the visual area at any one location has something to do with the next step, but the definition of the chain is configurational. This can be appreciated when the output of the different analyses methods in the experiments are correlated against the agent model, as shown in table 2. In each case a $t$-test was performed as a crude check of independence of the datasets. From the table, the isovist area is obviously associated with the agent result to a substantial degree, but the visual integration is not. This is perhaps to be expected given our discussion, but it is then surprising that the agent model correlates well with axial-line integration. If we were expecting the results to be

Table 2. Correlation coefficient $R^{2}$ and $t$-test $t$-statistic for the agent model data values against other visibility and spatial analysis techniques. In all cases $p<0.001$.

\begin{tabular}{llc}
\hline Visibility-analysis model & $\begin{array}{l}\text { Correlation with } \\
\text { agent model } R^{2}\end{array}$ & $\begin{array}{l}t \text {-test with } \\
\text { agent model } t \text {-statistic }\end{array}$ \\
\hline Isovist area (roads) & 0.59 & 8.3 \\
Visual integration (roads) & 0.36 & -16.5 \\
Axial-line integration & 0.66 & 26.3
\end{tabular}

(6) The original Space Syntax Laboratory study (1996) quotes $R^{2}=0.73$. However, this figure is achieved only if the values are averaged along lines. In order to correlate properly, all data points should be used individually, which yields the $R^{2}=0.66$ value reported herein. 
a direct consequence of our static visual analysis - as we might, as there is a visibility graph underlying both our VGA experiments and the agent model-then this order of correlation seems unlikely. In theory, it would seem, the axial-line analysis is the least related to the agents. Indeed, axial-line integration is often criticised as the construction of axial lines depends on the production of a set of convex spaces, and there is, in general, no single partitioning scheme to derive convex spaces (see, for example, Batty, 2001). Therefore, it would appear that the researcher must make a subjective decision on which lines to include, in turn, bringing his or her cultural background into the analysis. The agents, by contrast, have been implemented in a manner in which a strict, formal, sampling of the system is used. We should be wary that there are a number of factors that have crept into the agent analysis, in particular the release-zone area, and also the performing of an analysis on a current Ordnance Survey map that may not represent properly the actual ground-level features present when the analysis was performed several years ago. However, these, if anything, would lead us to expect less of a correspondence with axial-line integration. Therefore, it seems to me that the crucial factor at work is that both axial-line integration and the agent model are configurational samplers. There is a process at work in the construction of the axial map that is repeated in the agent analysis. In an urban situation such as this, the axial map approximates the topological relationships in the system. Indeed, current research into fractional analysis (Dalton, 2001), where the mean depth is weighted by the angle of incidence of the axial lines, has shown similar but improved results over standard axialline integration. More importantly to us, it is a purer topological sampler of a street system. Because the angular change is the important factor, the length of the line is no longer an issue (Turner, 2001). A road can therefore be split into many shorter segments, or even road centrelines, a problem which would previously have resulted in a dramatic shift in integration values. The researcher's own cultural input is essentially negated in systems where movement is directed along topological pathways, such as the urban study presented here, and the fractional-analysis approach approximates a topological description of the morphology. In a similar manner, the agents themselves move along and sample topological pathways as they choose locations stochastically in a forward direction. In a network of roads, where most locations lie ahead along the topology of the streets, the agents will choose in general to move along roads, turning into others when they find them - that is, at nodes on the topological graph. Thus, in the sense that the agents are analytic Markov samplers of the configuration, the individual step size becomes irrelevant to the agents: so long as each one is assumed to travel the distance between nodes taking up time from its journey, they might equally be applied to a visibility graph of topological nodes in a system of narrow streets, such as the point-based space-syntax graph proposed by Jiang and Claramunt (2002).

That topology may play an important part in the dynamics of actual urban systems is further underlined if we apply a multivariate regression of agents counts and axialline integration against the observed pedestrian gate counts. Doing so (see table 1), we find both an improved correlation coefficient and that the axial-line integration and agents have roughly equal input to this result (as evidenced by the $t$-ratios). That is, it appears that the axial-line integration is providing something extra that is not accessible to the agents, and vice versa. If we consider the axial lines as a topological system, they are providing a measure (in terms of fractional analysis) of the onedimensional routes from the current location to all topologically significant locations in terms (of fractional analysis) of their angular separation. We should be wary that there is a single result presented here, and that in an urban system we would expect (at least from our own cultural understanding) routes to be linearised around the topology of the system; however, understanding configuration in these terms leads to a natural 
system of cognition where a map can be represented as a one-dimensional set of relationships (Kuipers, 2000), which mirrors Penn's (2001) argument that the 'line' is the key to understanding the spatial morphology. The result of this discussion is that we have arrived at Hillier's (1996) statement that space itself is the machine. What the agents provide in terms of this machine are the cogs: the access to the machine at a physical level where one is required to walk (or transfer at some cost) from A to B, rather than transfer at the metalevel of topological nodes, and where one is required to see possibilities for this transfer at the level of the occupant of the system. That is, agents can provide the visual dynamics of the spatial morphology.

\section{Conclusion}

In this paper I have presented a review of visibility-analysis techniques applied to architecture and urban environments. The originators of the techniques cited Gibson's (1979) theory of direct perception as one of the prime motivations to develop analysis. Direct perception regards the relationship between the occupant and the environment rather than attempting to gain access to the vernacular phenomenological idea of perception. Thus it allows objective statements to be made about human-spatial interaction in the environment without confounding spatial and social variables. This would appear to make it a useful tool for providing quantified statements about the social adequacy of projects. However, through concentrating only on the visual relationships rather than a strict interpretation of direct perception, visibility analysis has tended to assess the visual experience within the environment or to classify the spatial components thereof. Both applications are subject to postpositivist criticism: how space is classified depends on the cultural background of the researcher; and a phenomenological account of space depends on the subjective experience of the occupant of an environment. To avoid these shortcomings, I propose that visibility analysis should assess not the visibility alone, but the visual ecological process which occurs between occupant and space. It has been shown that a process model can be constructed where sociological relationships are abstracted upwards from a layer of individuals, so that the physical relationships between aggregate behaviour and the environment can be investigated as a natural phenomenon, without individuation in the different languages of spatial and social variables. I have argued that agents employing an exosomatic visual architecture (University College London, 2000) approximate the process model. These agents were applied to a $1.5 \mathrm{~km} \times 1.5 \mathrm{~km}$ segment of an urban environment, and good correlation $\left(R^{2}=0.67\right)$ with observed numbers of pedestrians crossing seventy-eight 'gates' was discovered, provided the agents were released from specific subregions of the analysis area. The result corroborates an earlier finding of good correlation $\left(R^{2}=0.77\right)$ with human behaviour in a building environment (Turner and Penn, 2002).

Through a comparison of various different analysis techniques, the importance of the agents as samplers of the visual configuration has been demonstrated. Further, I have argued that both the agents and axial-line integration (Hillier and Hanson, 1984) should be regarded analytically as a measure of the street topology in an urban system. This leads to the view that the process of visual inhabitation is one driven by onedimensional routes through the configuration, and that the agents represent a means to assess the visual dynamics of the spatial morphology. The study presented in this paper represents a sample of ongoing work at University College London; whether or not agents are indeed topological samplers needs further research. However, the results seem to imply that a return to much simpler models of the environment may be fruitful: for example, it may be sensible to return to systems of axial lines as a basis for agent systems (Penn and Dalton, 1994), or sparse visibility graphs between topological nodes (Thomas and Donikian, 2000). Within building environments, of course, 
the topology is often not so rigorously defined as the means of movement between locations. In cases such as these, it may be worth considering other conceptualisations of the environment that could generate movement, for example, by examining recent geographic applications of surface networks (Rana and Morely, 2002) or Voronoï diagrams (Boots and Shiode, 2003).

Finally, it is time to reflect on the power of the process-based approach to inhabitation. Wheeler (1996) provides a philosophical account of the journey from animat research to the appreciation of Rothko's art, as in this paper I have provided a journey from visibility to visual inhabitation. Wheeler describes the interaction between the occupant and the environment (or 'viewer' and 'artwork') as a hermeneutical dialogue. A hermeneutical dialogue represents any interaction or relationship in the process of inhabitation, be it at the level of walking along a road or the enjoyment of an abstract painting. As such, the visual dynamic of spatial morphology is a dialogue in which a visual mirror is held to the phenomenological account of architecture in order to provide a logical account of the phenomena within architecture.

\section{References}

Amidon E L, Elsner G H, 1968, "Delineating landscape view areas: a computer approach", RN PSW-180, US Department of Agriculture, Washington, DC

Batty M, 2000, "Less is more, more is different: complexity, morphology, cities, and emergence" Environment and Planning B: Planning and Design $27167-168$

Batty M, 2001, "Exploring isovist fields: space and shape in architectural and urban morphology" Environment and Planning B: Planning and Design $28123-150$

Batty M, Jiang B, 1999, "Multi-agent simulation: new approaches to exploring space - time dynamics within GIS", WP 10, Centre for Advanced Spatial Analysis, University College London, London

Benedikt M L, 1979, “To take hold of space: isovists and isovist fields" Environment and Planning B: Planning and Design $647-65$

Benedikt M L, 1997, "Paths, people, and purposes, by Philip Thiel" ARCADE: The Journal for Architecture and Design in the Northwest 15(4) 14

Benedikt M L, Burnham C A, 1985, "Perceiving architectural space: from optic rays to isovists", in Persistence and Change Eds W H Warren, R E Shaw (Lawrence Erlbaum Associates, London) pp $103-114$

Boots B, Shiode N, 2003, "Recursive Voronoi diagrams" Environment and Planning B: Planning and Design $30113-124$

Braaksma J P, Cook W J, 1980, "Human orientation in transportation terminals" Transportation Engineering Journal 106(TE2) 189-203

Brooks R A, 1991, "Intelligence without representation" Artificial Intelligence 47139 - 159

Conroy R A, 2001 Spatial Navigation in Immersive Virtual Environments PhD thesis, Bartlett School of Graduate Studies, University College London, London

Dale K, Collett T S, 2001, "Using artificial evolution and selection to model insect navigation" Current Biology $111305-1316$

Dalton N, 2001, "Fractional configurational analysis and a solution to the Manhattan problem", in Proceedings of the 3rd International Symposium on Space Syntax Georgia Institute of Technology, Atlanta, GA, pp $26.1-26.13$

Davis L S, Benedikt M L, 1979, "Computational models of space: isovists and isovist fields" Computer Graphics and Image Processing 11(3) 49-72

De Floriani L, Marzano P, Puppo E, 1994, "Line-of-sight communication on terrain models" International Journal of Geographical Information Systems 8329 - 342

Desyllas J, Duxbury E, 2001, "Axial maps and visibility graph analysis", in Proceedings of the 3rd International Symposium on Space Syntax Georgia Institute of Technology, Atlanta, Georgia, pp $27.1-27.13$

Fisher P F, 1991, "First experiments in viewshed uncertainty: the accuracy of the view-shed area" Photogrammetric Engineering and Remote Sensing 571321 - 1327

Fisher P F, 1995, "An exploration of probable viewsheds in landscape planning" Environment and Planning B: Planning and Design $22527-546$

Fisher-Gewirtzman D, Wagner I A, 2002, "Spatial openness as a practical metric for evaluating built-up environments" Environment and Planning B: Planning and Design 3037 -49 
Gibson J J, 1950 The Perception of the Visual World (Houghton Mifflin, Boston, MA)

Gibson J J, 1979 The Ecological Approach to Visual Perception (Houghton Mifflin, Boston, MA)

Hanson J, 1994, “'Deconstructing' architects' houses” Environment and Planning B: Planning and Design 21675 - 704

Harvey D, 1973 Social Justice and the City (Edward Arnold, London)

Harvey I, Husbands P, Cliff D, Thompson A, Jakobi N, 1997, "Evolutionary robotics: the Sussex approach" Robotics and Autonomous Systems $20205-224$

Hillier B, 1996 Space is the Machine (Cambridge University Press, Cambridge)

Hillier B, Hanson J, 1984 The Social Logic of Space (Cambridge University Press, Cambridge)

Hillier B, Penn A, Hanson J, Grajewski T, Xu J, 1993, "Natural movement: or, configuration and attraction in urban pedestrian movement" Environment and Planning B: Planning and Design $2029-66$

Jiang B, Claramunt C, 2002, "Integration of space syntax into GIS: new perspectives for urban morphology" Transactions in GIS 295 - 309

Krüger M J T, 1979, "An approach to built-form connectivity at an urban scale: system description and its representation" Environment and Planning B: Planning and Design 6 67-88

Kuipers B, 2000, "The spatial semantic hierarchy" Artificial Intelligence 119191 - 233

Lee J, Stucky D, 1998, "On applying viewshed analysis for determining least-cost paths on digital elevation models" International Journal of Geographical Information Systems 12891 - 905

Llobera M, 1996, "Exploring the topography of mind: GIS, social space and archaeology" Antiquity $70612-622$

Lynch K, 1960 The Image of the City (MIT Press, Cambridge, MA)

Lynch K, 1976 Managing the Sense of Region (MIT Press, Cambridge, MA)

March L, Steadman P, 1971 The Geometry of Environment (Methuen, London)

Maturana H R, Varela F J, 1980 Autopoiesis and Cognition: The Realization of the Living (D Reidel, Dordrecht)

Maturana H R,Varela F J, 1987 The Tree of Knowledge: The Biological Roots of Human Understanding (Shambhala Publications, Boston, MA)

Oreskes N, Shrader-Frechette K, Belitz K, 1994, "Verification, validation, and confirmation of numerical models in the earth sciences" Science $263641-646$

O'Rourke J, 1987 Art Gallery Theorems and Algorithms (Oxford University Press, New York)

Penn A, 2001, "Space syntax and spatial cognition, or why the axial line?", in Proceedings of the 3rd International Symposium on Space Syntax Georgia Institute of Technology, Atlanta, GA, pp $11.1-11.16$

Penn A, Dalton N, 1994, "The architecture of society: stochastic simulation of urban movement", in Simulating Societies: The Computer Simulation of Social Phenomena Eds N Gilbert, J Doran (UCL Press, London) pp $85-125$

Penn A, Turner A, 2002, "Space syntax based agent models", in Pedestrian and Evacuation Dynamics Eds M Schreckenberg, S Sharma (Springer, Heidelberg) pp 99-114

Penn A, Conroy R, Dalton N, Dekker L, Mottram C, Turner A, 1997, "Intelligent architecture: new tools for the three dimensional analysis of space and built form", in Proceedings of the 1st International Symposium on Space Syntax University College London, London, pp 30.1-30.19

Peponis J, Wineman J, Rashid M, Hong Kim S, Bafna S, 1997, “On the description of shape and spatial configuration inside buildings: convex partitions and their local properties"

Environment and Planning B: Planning and Design $24761-781$

Rana S, Morely J, 2002, "Surface networks", WP 42, Centre for Advanced Spatial Analysis, Department of Architecture, University College London, London

Ratti C, 2002 Urban Analysis for Environmental Prediction PhD thesis, University of Cambridge, Cambridge

Soja E, 2001, "In different spaces", in Proceedings of the 3rd International Symposium on Space Syntax Georgia Institute of Technology, Atlanta, GA, pp s1.1 - s1.7

Space Syntax Laboratory, 1996, "Baltic House: preliminary report", technical report, Bartlett School of Graduate Studies, University College London, London

Teller J, 2003, "A spherical metric for the field-oriented analysis of complex urban open spaces" Environment and Planning B: Planning and Design $30339-356$

Thiel P, 1961, "A sequence experience notation for architectural and urban space" Town Planning Review $3233-52$

Thiel P, 1998, "People, Paths, and Purposes: Notations for a Participatory Envirotecture (University of Washington Press, Seattle, WA) 
Thomas G, Donikian S, 2000, "Modelling virtual cities dedicated to behavioural animation" Computer Graphics Forum 19(3) C71 - C80

Turner A, 2001, "Angular analysis", in Proceedings of the 3rd International Symposium on Space Syntax Georgia Institute of Technology, Atlanta, GA, pp 30.1 - 30.11

Turner A, Penn A, 1999, "Making isovists syntatic: isovist integration analysis", in Proceedings of the 2nd International Symposium on Space Syntax Volume III Universidad de Brasil, Brasilia, Brazil, 01.01 to 01.09, http://www.vr.ucl.ac.uktformat/research/vga/isss.pdf

Turner A, Penn A, 2002, "Encoding natural movement as an agent-based system: an investigation into human pedestrian behaviour in the built environment" Environment and Planning B: Planning and Design $29473-490$

Turner A, Doxa M, O'Sullivan D, Penn A, 2001, "From isovists to visibility graphs: a methodology for the analysis of architectural space" Environment and Planning B: Planning and Design $\mathbf{2 8}$ $103-121$

University College London, 2000, "A system and method for intelligent modelling of public spaces”, International Patent Application PCT/GB01/03763, European Patent Office, filed 2000

Wheeler M, 1996, "From robots to Rothko: the bringing forth of worlds", in The Philosophy of Artificial Life Ed. M A Boden (Oxford University Press, Oxford) pp 209-236 